\title{
Aux sources de la connaissance. L'état du débat constructivisme/ nativisme chez le très jeune enfant
}

parRoger Lécuyer [1]

etKarine Durand [2]

\section{Raccourcis}

- Résumé

- $\quad$ Plan de l'article

- $\quad$ Pour citer cet article

\section{Voir aussi}

- $\quad$ Sur un sujet proche

\section{Devenir}

2012/3 (Vol. 24)

- Pages : 82

- DOI : $10.3917 /$ dev.123.0181

- Éditeur : Médecine \& Hygiène

- À propos de cette revue

- Site internet

\section{Alertes e-mail}

Veuillez indiquer votre adresse e-mail pour recevoir une alerte sur les parutions de cette revue.

$\underline{\text { Voir un exemple }}$

Pages $181-214 \underline{\text { Article suivant }}$

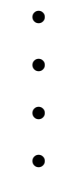




\section{Les premières connaissances : une place à part en psychologie?}

La psychologie cognitive du nourrisson a longtemps tenu, et continue dans une certaine mesure de tenir une place à part, en marge de la psychologie du développement. Les raisons de cette spécificité sont d'abord méthodologiques. Jusqu'à la fin des années 1950, la seule méthode disponible pour étudier le fonctionnement cognitif des bébés était l'observation. Dans le meilleur des cas, par exemple avec Piaget, cette observation était intervenante, et permettait de tester des hypothèses, mais elle n'était pas standardisée. Il est usuel de dater des publications de Fantz (1958) et de Berlyne (1958) l'entrée du bébé dans le laboratoire, point de départ du développement de méthodes très largement spécifiques pour l'étude du fonctionnement perceptif, puis cognitif de cet être qui ne sait pas parler mais sait pleurer et dormir. La lourdeur de l'expérimentation avec les bébés, la nécessité d'améliorer les procédures expérimentales, les discussions sur les processus cognitifs impliqués ou non dans ces expériences et la légitimité des conclusions qui peuvent en être tirées ont accentué cet isolement.

\section{$\underline{2}$}

Le caractère très nouveau et souvent spectaculaire des données obtenues dans ces expériences a produit des changements scientifiques certains sur la psychologie du développement, remettant en cause des connaissances considérées comme bien établies et avec elles les théories classiques, en particulier le grand édifice théorique piagétien. Par conséquent, ceci a eu des effets sur la sociologie de la science, transformant les leaders de la recherche sur le nourrisson en vedettes, et les chercheurs de psychologie de l'enfant d'âge préscolaire ou scolaire en critiques, défenseurs d'une orthodoxie théorique et méthodologique, considérant lesdites vedettes comme des gourous. Ce paysage est important à prendre en compte, parce qu'il n'est sans doute pas étranger à l'existence de prises de positions théoriques quelque peu radicalisées, cognitivistes et nativistes, lesquelles ont en retour renforcé le splendide isolement de la psychologie des premiers mois de la vie, coupée de la psychologie du développement dans les années 1960 à 1990.

Nous reviendrons sur ces cadres théoriques et leur impact, mais pour autant, tous les chercheurs s'intéressant aux bébés n'ont pas voulu cet isolement. Un certain nombre a au contraire tenté de faire évoluer les cadres théoriques classiques, envisageant ainsi toujours la psychologie du nourrisson comme une partie de la psychologie du développement. Avec le recul du temps, c'est sans doute Pierre Mounoud $(1971,1976,1979)$ qui apparaît comme le pionnier dans cette démarche, visant à faire évoluer la théorie de Piaget pour la rendre compatible avec les données nouvelles de la psychologie du nourrisson. Dans une série d'articles qui sont loin d'avoir eu l'impact qu'ils méritaient, il expose un système dont la grande nouveauté par rapport à Piaget consiste à séparer les structures et les modes de codage. Les structures sous-jacentes nécessaires à l'acquisition des connaissances ne sont pas comme chez Piaget construites par des restructurations successives dans le passage d'un stade à un autre : elles sont innées. Mais au fur et à mesure du développement se produisent des 
« révolutions » dans les systèmes de codage des caractéristiques de l'environnement. Le nouveau-né a un système de codage sensorimoteur, auquel correspondent une organisation interne, une structure sensorimotrice où les réflexes jouent un rôle important. Entre trois et six mois, il passe à un système de codage perceptivo-moteur et une organisation interne perceptivo-motrice se substitue progressivement à la précédente. Enfin, vers dix-huit mois, il y a passage du perceptivo-moteur au conceptuo-moteur et nouvelle réorganisation. Ces changements modifient profondément les modes de relation du bébé avec son environnement et, à chaque passage, se produit une réorganisation de l'ensemble de ces modes de relation. Les changements d'appellation correspondent à des modes de plus en plus complexes de relation avec cet environnement, même si la fonction de la composante motrice semble rester la même.

\section{$\underline{4}$}

L'un des apports importants de la théorie de Mounoud est d'expliquer les baisses d'efficience, et les disparitions de comportements (ce que l'on a longtemps appelé les courbes en U). Mais en même temps, c'est la faiblesse de son argumentation : en effet, ces régressions ne sont pas la règle mais l'exception dans le développement, et à chaque fois que l'on en observe, une composante exécutive motrice est en jeu. Or, deux spécificités du jeune être humain doivent absolument être prises en compte dans la manière d'envisager son développement. La première, bien connue depuis longtemps, est que comparée à la plupart des autres mammifères, l'espèce humaine est très peu performante d'un point de vue moteur pendant la période néonatale. La seconde, découverte plus récemment est que, au contraire, ce mammifère est en avance sur les autres en matière de développement sensoriel, dès la période fœtale (Granier-Deferre, Schaal et DeCasper, 2004). Les systèmes de codage hiérarchisés proposés par Mounoud ont tous en commun d'être moteurs, alors que c'est dans la motricité et l'efficience du corps propre qu'est le problème. On pourrait donc objecter à Mounoud un point sur lequel insiste Bullinger (1982) : "L'organisme est objet du milieu et pour les activités cognitives du niveau sensorimoteur c'est un objet à connaître ». Tant que l'on ne connaît pas encore l'outil qui va servir à connaître le monde, on peut difficilement connaître ce monde.

\section{$\underline{5}$}

La théorie de Mounoud a sans aucun doute le grand mérite de tenter d'adapter Piaget aux données nouvelles, mais en continuant à donner la même importance aux relations entre le perceptif et le moteur, elle ne permet pas de rendre compte des connaissances les plus précoces. Tenir compte du retard moteur et de l'avance perceptive de l'espèce a au contraire conduit indépendamment Mandler (1988) et Lécuyer (1989b) à proposer un autre cadre théorique. Ce point de vue a été repris et développé par la suite dans une série d'articles : Mandler (1992, 1998) ; Lécuyer (1994a, 1994b, 1996, 2000, 2001, 2004, 2011 ; Lécuyer, Rivière et Durand, 2006). La démarche adoptée se place dans une perspective écologique : elle ne part pas des seules données du laboratoire, mais se centre aussi sur les conditions dans lesquelles le bébé, dans son environnement quotidien, peut acquérir, développer, maîtriser des connaissances, y compris des connaissances de lois, en fonction de ses interactions avec le milieu physique et social. Ceci suppose que les activités perceptives du nourrisson s'exercent, dans ce milieu, dans des conditions qui leur permettent une certaine efficacité, et suppose également que le milieu usuel de vie fournit les stimulations nécessaires au développement cognitif, ce qu'il est évidemment nécessaire de montrer. 
Préalable à l'exposé de ce point de vue, sur les relations entre intelligence, perception et motricité, il est nécessaire de rappeler le rôle important joué par la perception dans la théorie de Piaget, car ce rôle est souvent sous-estimé. Si la motricité permet de produire les variations dans le milieu, qui sont la source de la connaissance, la perception et plus précisément la perception visuelle est le moyen de constater le résultat de l'action motrice et constitue donc l'acte même de connaissance. Une conception perceptive de l'intelligence visant à expliquer les connaissances précoces peut se situer dans une perspective néoconstructiviste en proposant un autre mode de relation entre la source de variation et l'analyseur des résultats produits. Puisqu'il s'agit d'expliquer l'existence de connaissances plus précoces que ne le supposait la théorie de Piaget, ces connaissances ne peuvent résulter de l'action du sujet, trop inefficiente. Cependant, la perception peut jouer le même rôle de constat que lui attribuait Piaget. Ainsi, Lécuyer (1989b) insiste sur le fait que si l'habituation est la technique de recherche de laboratoire dont l'efficacité est aussi remarquable, cela implique qu'elle s'exerce quotidiennement dans la vie du bébé. Il convient en effet de ne jamais oublier que le bébé ne participe à une expérience que si elle lui convient et que par conséquent, les comportements observés en laboratoire ont un rapport étroit avec ceux qui sont en œuvre dans la vie quotidienne. Lécuyer propose donc l'idée qu'avant le bébé physicien de Piaget, qui expérimente pour connaître les lois qui régissent les relations entre objets, existe un bébé astronome qui observe l'effet des variations de l'environnement qu'il n'a pas lui-même produites.

\section{$\underline{7}$}

Une objection peut être faite à un tel point de vue : les systèmes causaux en jeu dans l'environnement du bébé produisent des effets d'une manière souvent rapide, à des moments qui ne sont pas toujours prévisibles. L'apprentissage de lois d'une manière purement perceptive supposerait des capacités attentionnelles très importantes pour que soient saisies toutes les chaînes causales, condition nécessaire à l'apprentissage de lois. En d'autres termes, l'expérimentation, telle que l'envisage Piaget, est plus efficace que l'observation pour comprendre le fonctionnement de l'environnement. Le poids de l'argument se mesure au fait que la psychologie cognitive du nourrisson a pu se développer considérablement à partir de l'entrée du bébé dans le laboratoire. Effectivement, des apprentissages perceptifs sont possibles dans ce laboratoire, précisément parce que les conditions de présentation des événements sont placées sous le contrôle attentionnel des bébés. Toutefois, cette objection suppose que le bébé a, comme source essentielle d'information sur le monde physique, les transformations qui se produisent dans ce monde physique. Or ce n'est pas ce qui se passe.

Si l'on essaie de dégager les règles qui régissent les temps de regard des bébés sur ce qui se passe autour d'eux, on constate que les facteurs déterminants sont : 1) le mouvement ;2) la structuration ; 3) la nouveauté et 4) et à partir d'un certain niveau de connaissance, l'étrangeté. A ces propriétés des stimulations visuelles, il faut ajouter la multimodalité : dès la naissance, un contexte multisensoriel favorise davantage les prises de connaissance du bébé qu'un contexte unisensoriel (Bahrick, Flom et Hernandez-Reif, 2005 ; Bahrick, Lickliter et Flom, 2004). Or, dans l'environnement quotidien du bébé, ce qui bouge est le plus souvent humain ou animé par des humains ; ce qui est placé dans des conditions de distance permettant d'en saisir la structure est typiquement un visage humain ; ce qui est très souvent 
nouveau par l'angle de vue où les couleurs portées est humain; ce qui est étrange est souvent produit par l'humain dans des situations de jeu, et si bien des objets sont plus ou moins plurimodaux, rien n'est aussi multimodal qu'une mère.

Concernant la notion d'étrangeté présentée ci-dessus, elle fait référence aux réactions observées dans le paradigme usuellement appelé « de la transgression des attentes ». Pour les inventeurs du paradigme, une telle « attente » est déterminée par la connaissance d'une loi, d'une règle ou d'un principe [3][3] Ces trois termes sont utilisés dans la littérature.... En effet, dans ces situations, un bébé regarde plus longtemps un événement « impossible » qu'un événement " possible », ce qui conduit le chercheur à inférer qu'il n'attendait pas cet événement et donc qu'il connaît la loi physique qui détermine cette impossibilité. De fait, le sujet regarde plus l'événement que l'adulte juge impossible, mais ceci n'implique pas nécessairement qu'il y a pour lui violation d'une loi qui pourrait être décrite par : « les événements ne doivent pas se produire ainsi ». Il pourrait s'agir simplement d'une régularité : « habituellement, les événements ne se produisent pas ainsi ». La nature de l'attente peut donc être soit liée à la connaissance d'un « principe », qui dans une perspective nativiste serait complètement indépendant de l'expérience, soit liée à l'expérience antérieure sur le même type de situation. Dans le premier cas, la durée de regard serait déterminée par le caractère étrange, parce que contraire à la loi, de l'événement. Dans le second cas, il s'agirait bel et bien d'une réaction à la nouveauté, non pas par rapport à la phase d'habituation, comme on a parfois tenté de le démontrer (Bogartz, Shinskey et Schilling, 2000 ; Cashon, et Cohen, 2000 ; Schilling, 2000), mais par rapport à l'expérience quotidienne. Dans l'état actuel de nos connaissances, il est méthodologiquement impossible de décider entre ces deux interprétations.

Depuis la fin des années 1980, le point de vue selon lequel les connaissances sont acquises grâce aux interactions avec l'environnement, conformément à la pensée de Piaget, a continué à être exposé mais n'a connu qu'un succès très limité face au nativisme, prônant au contraire l'idée que les premières connaissances sont indépendantes de toute forme d'apprentissage (Spelke, 1998). Dans la mesure où les preuves de l'existence de ces connaissances ne sont présentes qu'après deux mois, il est possible d'imaginer que dans les premiers mois ces connaissances fassent l'objet d'apprentissages. Cette hypothèse est d'autant plus plausible qu'a été démontrée la possibilité d'apprentissages perceptifs très rapides, pour peu que les bébés soient placés dans de bonnes conditions (Baillargeon, 2004).

\section{$\underline{11}$}

Il est donc nécessaire de se demander pourquoi le nativisme reste prépondérant. Une raison se situe sans doute dans la dissymétrie des deux hypothèses. En effet, envisager qu'une connaissance, mise en évidence à un âge précoce, est innée est à première vue une explication qui s'auto-suffit. Envisager au contraire qu'elle fait l'objet d'un apprentissage nécessite de fournir des détails sur la manière dont cet apprentissage s'opère. Il y aurait sans doute à dire au sujet de cette dissymétrie et de cette autosuffisance, mais dans un premier temps, nous les prendrons en compte. Dans le cadre d'une conception perceptive du développement cognitif, expliquer la présence de connaissances précoces suppose une description des mécanismes d'apprentissage en jeu, des niveaux de représentation nécessaires, des modes de passage d'un 
niveau au niveau supérieur, des pré-requis nécessaires à ces formes d'organisation cognitive. Or, toutes ces propositions ne figuraient pas dans les conceptions néoconstructivistes des années 1990. C'est sans doute ce qui a laissé le champ libre au nativisme. C'est aussi ce qui nécessite un réexamen de ces questions théoriques.

\section{Peut-on ne pas être nativiste?}

Le mot nativisme, et celui d'innéisme sont souvent considérés de manière équivalente ou pour le moins partiellement confondue. Il est donc nécessaire de préciser ici de quoi l'on parle. Il existe une fameuse querelle de l'inné et de l'acquis, dont les origines se perdent dans les débuts de la réflexion philosophique et dont Lemaine et Matalon (1985) ont bien montré qu'elle a peu de chances de trouver une fin, bien qu'étant régulièrement présentée comme dépassée. Cette querelle oppose l'innéisme classique à l'environnementalisme non moins classique et l'objet du débat est l'explication des différences individuelles. Depuis la regrettable invention du QI, celui-ci focalise particulièrement la dispute, et il s'agit typiquement de savoir si la différence entre un QI de 90 et un de 110 est innée ou bien acquise, ou bien, variante moins rigide, de savoir ce que cette différence doit aux gènes et à l'environnement.

\section{$\underline{13}$}

Contrairement à l'innéisme classique, le nativisme ne s'intéresse nullement aux différences individuelles, mais cherche à expliquer l'existence des connaissances précoces. Prenons dans un premier temps le fameux exemple de la permanence de l'objet. Assumons provisoirement qu'il est démontré que cette permanence a été mise en évidence à 2,5 mois (Aguiar et Baillargeon, 1999). Elle n'est pas explicable dans le cadre de la théorie de Piaget, puisque celle-ci suppose que la permanence se construit sur la base de l'action sensorimotrice du sujet, et qu'une condition nécessaire (mais non suffisante) à l'efficience de cette action est la coordination entre vision et préhension, qui n'apparaît qu'à cinq mois. En conséquence, la permanence se développe lentement et est inexistante avant neuf mois, puisque jusque-là, les bébés ne recherchent pas les objets disparus sous leurs yeux. La conclusion de la théorie nativiste est fort simple : la permanence de l'objet n'est donc pas acquise par les mécanismes décrits par Piaget. Elle est donc innée. Plus généralement, il existe un noyau initial de connaissances présent dès la naissance, et sur lequel vont pouvoir se greffer les apprentissages ultérieurs. C'est le « core knowledge » de Spelke (1998).

\section{$\underline{14}$}

Une version plus extrémiste du nativisme avait été présentée auparavant par Mehler (1983; Mehler et Dupoux, 1990). "Un sujet ne peut apprendre de concept nouveau, s'il ne possède pas déjà les bases conceptuelles requises pour formuler les hypothèses nécessaires à sa confirmation. Il y a donc impossibilité d'apprendre ce qu'on ne possède pas déjà sous une forme ou sous une autre » (Mehler, 1983, pp. 133). La comparaison entre « l'état initial» des capacités cognitives du nouveau-né et «l'état stable » des capacités de l'adulte montre donc beaucoup de points communs. Ceci au passage rend superfétatoire la psychologie du développement qui ne peut servir qu'à décrire des changements complètement secondaires, puisque fondamentalement, rien ne change. 
Dans cette version extrême, et surtout dans la version plus nuancée de Spelke, le nativisme a été depuis cinquante ans la théorie dominante en psychologie cognitive du nourrisson. Il a dû pour cela résister à un certain nombre de critiques. Citons-en quelques-unes : sur la permanence de l'objet, les piagétiens ont demandé qu'on leur explique pourquoi les bébés de sept mois ne recherchent pas un objet qui vient de disparaitre sous leurs yeux. La permanence de Baillargeon est-elle aussi solide que celle de Piaget ? La réponse à cette question est généralement fournie en termes de fonctions exécutives : ce n'est pas parce que l'on sait qu'un objet disparu continue d'exister que l'on sait aller le chercher, même si l'on sait effectuer séparément toutes les actions nécessaires à cette recherche. Ainsi, si les bébés de neuf mois savent rechercher un objet caché, ils commettent une erreur dans cette recherche si l'on change l'endroit où l'objet est caché : ils retournent le chercher là où ils l'avaient trouvé antérieurement. C'est ce que Piaget appelait l'erreur du stade 4 et que l'on appelle usuellement l'erreur A non B. Piaget y voyait un défaut de permanence, mais des erreurs de recherche peuvent être mises en évidence avec des enfants bien plus vieux, et ayant acquis la permanence de l'objet du point de vue piagétien même. Ainsi, Rivière et Lécuyer (2003) ont montré des erreurs de recherche chez des enfants de 30 mois. Sur la permanence de l'objet toujours, l'expérience princeps de Baillargeon, Spelke et Wasserman (1985) a fait l'objet d'un acharnement remarquable dans la critique méthodologique (Bogartz, Shinskey et Schilling, 2000 ; Cashon et Cohen, 2000 ; Schilling, 2000), mais la permanence précoce a été retrouvée maintes fois avec des procédures expérimentales différentes, dans des laboratoires différents. Pour autant, toutes les capacités que la recherche nativiste a cru mettre en évidence n'ont pas été retrouvées, et il existe même des contre-exemples fameux, comme les capacités " numériques » précoces qui de fait ne sont pas numériques, mais sont des capacités de différenciation de quantités discrètes ou de repérages spatiaux et temporels efficaces (Lécuyer, 2002). Globalement, comme c'est le cas pour cet exemple, c'est beaucoup plus sur l'interprétation que sur les faits eux-mêmes que les questions se posent.

Il faut en fait considérer que le nativisme a longtemps été dans l'air du temps, parce que compatible avec le cognitivisme dans sa forme computationnelle. $\mathrm{Si}$ « le cerveau est un ordinateur » et puisque les ordinateurs que nous savons fabriquer savent d'emblée faire des choses « intelligentes », alors le petit cerveau humain du bébé le peut aussi. Or le cognitivisme et le nativisme ont un point commun capital : la volonté d'ignorer le développement. Cette volonté est évidente pour le cognitivisme, puisque les ordinateurs ne se développent pas (côté matériel) et qu'il y a des développeurs pour les faire fonctionner (côté logiciel). Elle est moins évidente pour le nativisme, mais Jacques Mehler (1983, Mehler et Dupoux, 1990) explique qu'étudier le développement ne présente pas grand intérêt.

\section{$\underline{17}$}

La conséquence de ce qui précède est que jusque dans les années 1980/90, la psychologie cognitive du nourrisson est largement a-développementale. Mais les recherches se multiplient, et bien sûr, elles montrent que les performances cognitives ne sont pas les mêmes à trois mois qu'à six. Si donc on s'en tient aux faits mis en évidence, toujours dans le laboratoire, on assiste à un retour de ce domaine de recherche dans le cadre de la psychologie du développement. Ce retour n'est pas sans poser de problèmes théoriques : les alternatives au nativisme proposées antérieurement mais n'ayant eu qu'une audience minoritaire ne seraient- 
elles pas finalement plus adéquates pour rendre compte d'un certain nombre de faits? La conception piagétienne, un peu vite passée à la trappe par tous les spécialistes, n'aurait-elle pas elle aussi quelques mérites explicatifs à prendre en compte pour décrire le développement cognitif entre la fin de la vie intra-utérine et les débuts du langage? Le cognitivisme computationnel a depuis longtemps montré ses limites, et la majeure partie de ce que nous savons sur l'intelligence des bébés est basée sur les variations de leur attention, alors que l'importance des processus attentionnels dans le fonctionnement du cerveau humain le différencie de manière radicale d'un ordinateur, toujours prêt à recevoir l'information qui lui est fournie ... ou bien en panne.

\section{$\underline{18}$}

Rappelons pourtant, avant d'oublier la métaphore informatique que c'est dans le domaine de l'intelligence précoce que celle-ci a trouvé l'une de ses applications (Bornstein et Sigman, 1986 ; Lécuyer, 1989a) avec le raisonnement sous-jacent suivant : la durée d'habituation moyenne à un stimulus donné diminue avec l'âge et il existe des différences individuelles importantes dans la durée d'habituation de bébés du même âge au même stimulus. Ces différences de durée sont des différences de vitesse d'habituation, et résultent de différences de vitesse de traitement de l'information. Or l'intelligence, c'est-à-dire la puissance du processeur central, est la vitesse de traitement de l'information. Sur ces bases, on a cherché des corrélations entre d'une part les durées d'habituation et d'autres indices plus ou moins complexes tirés des situations d'habituation et, d'autre part, le QI ultérieur. Ces corrélations sont supérieures à celles obtenues à partir des tests pour bébés, mais restent trop faibles pour des prédictions individuelles (0,37 dans une méta-analyse de Kavsek, 2004). Néanmoins, sur cette base, a été mis au point le Fagan test of infant intelligence qui n'a toutefois connu qu'un succès marginal (Fagan, 2007).

La démarche de Fagan et celle de Mehler ne se confondent en aucun cas : le premier se centre sur les différences individuelles que le second ignore volontairement, mais ils ont en commun un fort déterminisme : pour le premier, les variations observées dans les durées d'habituation ne doivent que très peu aux variations intra-individuelles dans les performances, ce qui concernant les bébés est une hypothèse très forte : en fait, les corrélations de type test/retest dans les situations d'habituation sont généralement faibles (Lécuyer, 1989a ; Lécuyer et Streri, 1986). Pour le second, un système de connaissances est organisé à la naissance, indépendamment de toute interaction avec l'environnement sur lequel portent ces connaissances. Le premier reprend la conception de Gesell qui réduit le développement à la maturation, le second attribue au développement une place négligeable, puisqu'il parle de connaissances avant l'apprentissage (Mehler, 1983).

\section{$\underline{20}$}

S'il a le mérite d'être aussi spectaculaire que contre-intuitif, le nativisme souffre de deux difficultés majeures : la première est liée au développement de nos connaissances sur les activités perceptives et cognitives du fœus. La naissance ne peut plus être considérée comme l'état initial de Mehler, pas plus que comme la table rase empiriste. L'existence d'apprentissages prénatals étant maintenant bien établie, il est clair que l'inné peut être acquis. 
La deuxième concerne le domaine de la connaissance étudié par ce courant. Comme Piaget, le nativisme s'est essentiellement attaché à fournir une explication des origines de la connaissance de la physique. Dans cette perspective, ce courant s'intéresse avant tout aux connaissances de lois qui définissent les contraintes de fonctionnement physique des objets grâce auxquelles le bébé raisonne conceptuellement (la pesanteur, la solidité, la continuité, etc.). Pour autant, aucune connaissance de loi n'a pu être mise en évidence avant l'âge postnatal de 2-3 mois. Le « core knowledge » est donc bien présent à trois mois, mais cet âge est bien tardif pour que les connaissances soient innées. De fait, la période qui va de deux à cinq mois environ se trouve sous les feux de la question théorique majeure de l'origine des connaissances de lois. En effet, il est trop tôt avant cinq mois pour que celles-ci soient issues d'apprentissages piagétiens, et il est trop tard à deux mois pour qu'elles soient «innées » au sens où l'entend le nativisme. Il semble donc nécessaire de chercher une autre solution théorique, et sans doute de revenir à des propositions néoconstructivistes. Avant d'examiner de plus près les conditions que devrait satisfaire un cadre théorique rendant compte de l'acquisition de ces connaissances de règles vers la fin du premier trimestre de vie postnatale, nous allons procéder à quelques rappels, d'une part sur les apprentissages prénatals, d'autre part sur les principales connaissances présentes à trois mois.

\section{L'apprentissage commence avant la naissance}

A partir des années 1970, les recherches sur le développement sensoriel du fœtus confirment l'existence d'un fonctionnement perceptif et cognitif présent dès avant la naissance : les différents systèmes sensoriels se développent pendant la période fœtale selon un ordre commun à tous les mammifères, allant dans le sens Toucher - Olfaction - Gustation Audition - Vision et, si l'on met à part la modalité visuelle, toutes les autres sont fonctionnelles au cours du troisième trimestre de vie (bien que non matures, la maturation sensorielle continue bien après la naissance). L'environnement perceptif du fotus est riche et varié en stimulations sensorielles : des informations tactiles et vestibulaires, olfactives et gustatives, sonores (et dans une moindre mesure visuelles) auxquelles le fotus peut être exposé de manière régulière. Les premières réactions fotales sont très précoces (vers 8-9 semaines) et sont des réponses aux stimulations tactiles. Plus tard, vers 25 semaines, le fotus commence à réagir aux odeurs et aux saveurs qui composent son liquide amniotique (Schaal, Hummel et Soussignan, 2004). Vers 30 semaines, il réagit à des bruits externes (GranierDeferre, Schaal et DeCasper, 2004). En fin de gestation, il peut réagir à des stimulations visuelles (Del Giudice, 2011). Pour des raisons méthodologiques et éthiques, les travaux menés dans ce domaine ont surtout interrogé les compétences auditives et olfactives du fœtus humain et les preuves de l'existence d'un fonctionnement perceptif mis en évidence par ces recherches constituent actuellement l'un des éléments les plus forts de remise en question des thèses nativistes. Les réponses comportementales du nouveau-né, parfois si surprenantes, trouvent dorénavant une explication dans les acquisitions réalisées pendant la période fœtale sous l'effet d'expériences sensorielles régulières qui laissent des traces en mémoire. En particulier, les préférences du nouveau-né humain, longtemps supposées innées faute de pouvoir les expliquer, paraissent être partiellement sous le contrôle des apprentissages prénatals. Decasper et Fifer (1980) observent par exemple que le nouveau-né préfère la voix maternelle à une autre voix féminine parce qu'il y a été exposé avant la naissance. Le fotus, 
régulièrement exposé aux sons de parole, apprend la prosodie et peut, après la naissance, reconnaître entre autres sa langue maternelle et la préférer à une langue étrangère (appartenant à une classe rythmique éloignée) ou encore une histoire familière plutôt que la même histoire dont certains mots-clés ont été remplacés par des nouveaux (De Casper et Spence, 1984). Dans le domaine olfactif, Schaal, et al. (Schaal, Marlier et Soussignan, 1995 ; Schaal, Marlier et Soussignan, 1998 ; Marlier et Schaal, 2005) montrent que les nouveau-nés reconnaissent et préfèrent une odeur à laquelle ils ont été exposés in utero : les bébés s'orientent davantage vers l'odeur de leur propre lait maternel ou encore vers l'odeur de leur propre liquide amniotique. L'explication de ces préférences olfactives serait à mettre en relation avec des composés chimiques partagés entre le liquide amniotique et le lait maternel. Une odeur peut être apprise avant la naissance : des fœtus exposés à l'odeur d'anis reconnaissent cette odeur après la naissance (Schaal, Marlier et Soussignan, 2000).

\section{$\underline{23}$}

Pour Schaal (2011), le nouveau-né serait en état « d'attente sensorielle », c'est-à-dire que ses conduites initiales seraient orientées par des éléments rencontrés in utero et intégrés par le système en développement. C'est pourquoi, certaines des caractéristiques de ces stimulations (la voix maternelle ou l'odeur du liquide amniotique par exemple), devenues familières, seront reconnues et préférées à la naissance. Qui plus est, cette base de familiarité, vers laquelle le nouveau-né se tourne, pourrait lui servir de guide et permettre ainsi la transition entre deux environnements et une adaptation plus souple au monde aérien. Cette notion d'attente sensorielle fournit une explication possible aux connaissances les plus précoces. Si certaines connaissances sont effectivement observées à la naissance, leur origine se trouve dans l'interaction entre les stimulations fournies par le milieu intra-uterin et les différents systèmes sensoriels qui les traitent. Ainsi, les études sur la période prénatale invitent à ne plus considérer la naissance comme le point de départ, l'état initial du développement cognitif. Les compétences néonatales et leurs explications potentielles justifient pleinement l'hypothèse selon laquelle " certains processus psychobiologiques commencent à fonctionner bien avant la naissance sur un mode analogue à celui de la période postnatale suggérant la possibilité d'une continuité transnatale », (Lecanuet, 1993, p. 33). Cette continuité transnatale est assurée par le développement en continu des systèmes sensoriels et les prises de connaissances du sujet interagissant avec son milieu.

\section{Les connaissances mises en évidence de deux à cinq mois sont-elles innées ?}

\section{$\underline{24}$}

Si l'on souhaite expliquer l'existence d'un certain nombre de connaissances dans les premiers mois de la vie, avant de savoir si leur origine se situe ou non dans des apprentissages, il est d'abord nécessaire de situer le moment où elles sont présentes, et quelles elles sont. Sur la première question, il est remarquable qu'aucune connaissance de règles n'ait pu être mise en évidence avant 2-3 mois. Sur la seconde, il n'est pas possible de présenter ici l'ensemble des connaissances présentes dans cette période de 2-5 mois, mais quelques exemples suffiront. 
Suivant parfaitement la problématique piagétienne, les nativistes s'intéressent principalement aux connaissances des règles de fonctionnement des objets amenant à la description d'un bébé physicien et rationnel. Le cœur du débat reste donc très piagétien : c'est l'objet envisagé dans ses caractéristiques physiques et spatiales (ses propriétés et sa permanence). Là peut-être plus qu'ailleurs, Piaget persiste et il reste difficile d'aborder le débat sans rappeler ce que l'on doit au pionnier de la psychologie du nourrisson : avoir posé toutes les bonnes questions.

\section{$\underline{26}$}

Cependant, aux antipodes de la conception piagétienne, les nativistes considèrent que le bébé possède une connaissance innée de l'objet : il sait que l'objet existe dans l'espace et le temps de façon permanente et peut ainsi prédire le déroulement d'événements impliquant des objets. L'hypothèse du concept d'objet inné est envisagée grâce à l'existence supposée de connaissances inscrites dans l'esprit humain dès la naissance (le core knowledge). Spelke (1994, 1998) insiste plus particulièrement sur deux principes innés : la continuité (l'objet existe de façon continue dans l'espace et dans le temps) et la solidité (deux objets ne peuvent pas occuper le même espace en même temps). Les nouveau-nés ne partent donc pas de rien puisqu'ils sont en quelque sorte guidés par ces deux principes dans leur compréhension du monde physique.

\section{$\underline{27}$}

Examinons plusieurs de ces connaissances supposées innées et tout d'abord l'incontournable permanence de l'objet. Ici encore, Piaget revient. Mais à la différence de Piaget qui interroge le bébé en lui proposant une recherche manuelle de l'objet caché, Baillargeon, et al. (1985) le questionnent à partir de situations visuelles d'un objet partiellement ou complètement caché. Dans la première expérience utilisant la méthode de la transgression des attentes, l'expérience du pont-levis, les auteurs constatent qu'à cinq mois les bébés savent qu'un objet disparu continue à exister. Des résultats similaires ont ensuite été retrouvés avec des bébés âgés de 2,5 mois (Aguiar et Baillargeon, 1999). Il est remarquable que sur cette question d'une importance théorique fondamentale et ayant fait l'objet d'un grand nombre de publications sur des âges supérieurs à deux mois, aucune publication n'ait à ce jour mis en évidence la permanence de l'objet à la naissance. Vers 3-4 mois, les bébés comprennent qu'un objet solide ne peut pas traverser (ou être traversé par) un autre objet solide (Hespos et Baillargeon, 2001). Ils savent également qu'un objet en mouvement partiellement caché derrière un bloc conserve son unité (Kellman et Spelke, 1983). La question de l'unité de l'objet a été posée à des nouveau-nés et les résultats montrent que cette connaissance n'est pas présente à la naissance (Slater, Morison, Somers et Mattock, 1990 ; Slater, Johnson, Kellman et Spelke, 1994)). L'unité de l'objet apparaît de fait vers deux mois (Johnson et Nañez, 1995). De toute évidence, les données empiriques sur la connaissance de l'objet et des lois qui les gouvernent, convergent sur un point : l'existence de connaissances n'est observée qu'à partir de 2-3 mois et pas dans la période qui précède. Ces connaissances sont-elles innées ? Si l'on tient compte du fait que le nourrisson humain est en avance sur les autres mammifères dans son développement sensoriel, il est tout à fait envisageable que des apprentissages perceptifs puissent être réalisés au cours des deux premiers mois de vie, apprentissages qui paraissent suffisants pour expliquer les connaissances observées à 2,5 mois, y compris celles relatives à la permanence et à l'unité de l'objet. Si ces connaissances ne sont pas innées, elles se développent progressivement. Elles deviennent de plus en plus nombreuses et complexes permettant ainsi au bébé de comprendre davantage de situations impliquant des objets. Le 
développement des connaissances sur les compétences du bébé impose de nouveau une perspective développementale.

\section{$\underline{28}$}

Le raisonnement du bébé ne fonctionne pas en tout ou rien et ce qui paraît être compris dans une situation ne l'est pas forcément dans une autre pourtant très proche. Prenons l'exemple de la compréhension du bébé de situations d'objets cachés : toutes les situations de disparition d'un objet ne sont pas comprises aux mêmes âges. Baillargeon (2004) a étudié précisément trois situations de disparition d'un grand objet : 1) l'occultation (un grand objet disparaît derrière un petit écran) ; 2) l'inclusion (un grand objet disparait dans un petit récipient) et 3) le recouvrement (un grand objet disparaît sous un petit couvercle). Si les bébés de 2,5 mois sont étonnés par la situation d'occultation, ce n'est que cinq mois plus tard qu'ils le sont dans la situation d'inclusion et il faut attendre environ dix mois de plus pour la situation de recouvrement. Ces expériences confirment que des situations perceptivement proches sont traitées de manière très différente par les bébés. Comment expliquer ces décalages importants ? Lécuyer (2004) propose l'hypothèse suivante : les décalages sont dus à la fréquence avec laquelle ces situations sont rencontrées quotidiennement par les bébés. Dans notre quotidien, les situations de disparition d'un objet qui passe derrière un autre objet sont beaucoup plus fréquentes que celles dans lesquelles l'objet disparaît sous un autre objet.

\section{$\underline{29}$}

L'expérience perceptive serait donc l'une des sources permettant au bébé d'apprendre à raisonner sur les principes spatiotemporels qui organisent le réel. Les connaissances de lois seraient dépendantes des interactions avec l'environnement et les événements qui s'y déroulent. Baillargeon (2004) constate que pour une même situation, les bébés apprennent à prendre en compte les différentes informations pertinentes dans la situation de manière progressive. Par exemple, alors que les bébés de 2,5 mois savent que l'objet disparu derrière un cache doit être invisible et que le cache doit être visible, ce n'est qu'un mois plus tard qu'ils comprennent qu'un objet plus haut que le cache doit être partiellement visible et cinq mois plus tard qu'ils comprennent que l'objet caché derrière un écran transparent reste visible. Il en va de même pour la connaissance du principe de gravité. A trois mois, les bébés semblent considérer que n'importe quel contact d'un objet avec un support empêche qu'il tombe, et il faut attendre l'âge de six mois pour qu'ils prennent en compte l'importance et la localisation du contact pour prédire à partir de quand l'objet doit ou ne doit pas tomber (Needham et Baillargeon, 1993).

\section{$\underline{30}$}

Prenons un autre exemple, les situations de catégorisation. Ce que l'on appelle catégorisation chez le bébé est usuellement opérationnalisé de la manière suivante : dans une phase d'habituation, on présente à chaque essai un stimulus différent, mais tous les stimuli sont différentiables par les bébés de l'âge considéré et appartiennent à la même catégorie. Dans la phase test, on présente un nouveau stimulus appartenant à la catégorie et un autre qui n'y appartient pas. On parle de catégorisation si le premier n'est pas traité comme nouveau (et donc il n'y a pas de remontée des temps de fixation) alors que le second l'est (il y a cette remontée). Les premières manifestations de catégorisation sont présentes vers trois mois (Lécuyer et Poirier, 1994 ; Poirier, Lécuyer et Cybula, 2000), même si les bébés sont capables 
de dégager un invariant perceptif simple plus tôt. En termes de représentation, la catégorisation pose plusieurs questions très importantes.

\section{$\underline{31}$}

Une première question est de savoir si cette catégorisation est d'abord perceptive puis peut devenir plus tard conceptuelle ou si les deux formes de catégorisation coexistent dès le départ, ce qui ne suppose pas les mêmes niveaux de représentation (Rakinson et Oakes, 2003). Par exemple, les bébés de 4-5 mois catégorisent les chiens et les chats (Eimas et Quinn, 1994 ; Eimas, Quinn et Cowan, 1994 ; Furrer et Younger, 2005), ou les chaises, les lits, les canapés et les tables (Behl-Chadha, 1996) mais avec des dissymétries : si on les habitue à des photos de chats, les bébés réagissent à la nouveauté pour un chien, mais la réciproque n'est pas vraie. Est-ce que le « concept» de chat est plus précoce que celui de chien ? Une explication plus simple est que la variété des formes de chien est très supérieure à celle des formes de chat, ce qui fait qu'un chat est compatible avec la catégorie chien alors que l'inverse n'est pas vrai. Si cette seconde explication est retenue, la catégorisation est perceptive, ce qui veut dire que les bébés classent des formes. C'est aussi ce qui se passe quand les bébés de cinq mois catégorisent les animaux et les meubles au vu d'une série de photos qui pour les unes comportent majoritairement des droites (meubles) et pour les autres ne comportent que des courbes (animaux). Une condition nécessaire pour pouvoir parler de concept est de démontrer que la différenciation des catégories peut se baser sur d'autres critères que la forme et par exemple l'attribution de capacités ou de fonctions, et ce n'est que bien plus tard que les bébés montrent qu'ils savent qu'un animal boit mais pas un véhicule (Rakinson et Oakes, 2003).

\section{$\underline{32}$}

Une autre manière d'aborder la même question consiste à se demander si les catégories testées en laboratoire ont été élaborées dans la vie quotidienne des bébés ou sont apprises dans les conditions à la fois bien contrôlées et tenant compte des capacités attentionnelles des bébés, dans le laboratoire. Poirier, Lécuyer et Cybula (2000) ont montré dès les années 1990 qu'une catégorie complètement artificielle pouvait être apprise dans le très court temps d'une expérience de laboratoire par des bébés de trois mois. Mais Bornstein et Masch (2010) ont comparé un groupe d'enfants familiarisés avec des objets complètement artificiels et inconnus de trois à cinq mois et des bébés n'ayant pas bénéficié de cette familiarisation et testés aussi à cinq mois. Si ces derniers explorent plus longtemps les objets appartenant à la catégorie, la différenciation des objets nouveaux, suivant qu'ils appartiennent ou non à cette catégorie, est la même dans les deux groupes. Il paraît difficile de penser en l'occurrence qu'un concept a été élaboré.

\section{$\underline{33}$}

Dernière question importante, du point de vue qui nous occupe : quelle forme peut bien prendre la représentation d'une catégorie ? Bornstein et Masch (2010) opposent deux hypothèses : ou bien chaque exemplaire est représenté en mémoire, ou bien un prototype, ou une loi générale l'est. Du point de vue des capacités cognitives nécessaires, dégager un prototype semble plus difficile, mais on sait que chez l'adulte, le codage de chaque exemplaire est rapidement limité par le nombre d'items. Tout porte à croire que cette limitation est plus rapide pour les bébés. La capacité à dégager un invariant pour l'ensemble des stimuli présentés s'impose donc (Younger, 2003 ; Poirier, 1995), ce qui fait de la 
catégorisation une capacité à tenir compte de dimensions qui étant dans tous les stimuli présentés ne sont dans aucun en particulier mais sont dans la relation entre ceux-ci.

L'existence d'apprentissages perceptifs de lois entre deux et cinq mois est donc bien démontrée. Les bébés peuvent apprendre une règle pendant les quelques minutes que dure une expérience, sur la base de quelques démonstrations (Baillargeon, 2004, pour une synthèse). L'apprentissage par observations semble être une source puissante dans la construction du réel.

\section{A quelles conditions un néoconstructivisme est-il envisageable?}

Le nativisme étant clairement en difficulté, et le retour de la psychologie cognitive du nourrisson dans un cadre développemental étant de plus en plus évident, la porte semble ouverte à une conception plus classique, tenant compte de tous les apports piagétiens les plus fondamentaux et donc à une forme de néoconstructivisme. Mais si, comme nous l'avons vu au début de cet article, les tenants de ce type d'approche n'ont pas convaincu, c'est que toutes les conditions n'étaient pas remplies pour que la valeur explicative de ce point de vue théorique soit suffisante. Il est donc nécessaire de revenir sur les conditions qui restent à remplir. Trois sont selon nous nécessaires : 1) il faut décrire des niveaux de représentation permettant de rendre compte de niveaux d'organisation des connaissances dans plusieurs domaines et les mécanismes d'apprentissage successivement en jeu lors du développement, et singulièrement ceux qui permettent d'acquérir des règles ; 2) il faut décrire les conditions nécessaires au passage d'un niveau de représentation au niveau supérieur ; 3) il faut prendre en compte l'importance fondamentale démontrée dans les années récentes de la multimodalité dans les mécanismes de prise d'information sur l'environnement.

\section{Des niveaux de représentation et des mécanismes d'apprentissage}

Si le terme de représentation a fait l'objet d'assez de polémiques pour que beaucoup d'auteurs l'évitent soigneusement, c'est avec comme raison principale qu'il manque de précision et que les mêmes réalités ne sont pas toujours situées derrière le même concept. C'est aussi, et les deux vont ensemble, parce que pour la représentation comme pour bien d'autres capacités, on a longtemps raisonné en tout ou rien, la représentation étant présente ou absente à tel ou tel âge chez le bébé. La tradition du débat sur ce point remonte à Wallon et Piaget, qui visiblement déjà ne parlaient pas de la même chose. Comme pour d'autres concepts aussi, la solution est sans doute à rechercher dans le développement progressif d'une capacité, donc dans la description par le chercheur de niveaux successifs de représentation permettant chacun 
de rendre compte de l'existence de compétences ou de connaissances qui comportent des contraintes cognitives jugées homologues.

\section{$\underline{37}$}

Lorsque cette homologie est avérée, ou pour le moins jugée très probable, la définition d'un niveau de représentation a pour avantage que le débat sur la réalité de telle ou telle connaissance chez le bébé ne se fait pas de manière isolée, savoir par savoir, mais de manière plus globale : par exemple, la capacité de catégoriser des objets n'implique-t-elle pas celle de percevoir l'unité des objets? La discussion risque moins ainsi de tomber dans un méthodologisme étroit et implique nécessairement à la fois des aspects théoriques et méthodologiques. C'est pourquoi, Durand, Lécuyer et Frichtel (2003) ont proposé un premier système hiérarchique de représentations. Celui-ci a été ensuite développé par Lécuyer (2011; cf. tableau I) pour la période qui va du début de la maturation des différents systèmes sensoriels à 2-3 mois, âge où sont mises en évidence les premières connaissances. Nous essaierons ici de préciser et de faire évoluer un peu plus ce système.

Tableau I - Les quatre niveaux de représentation proposés et les capacités qu'ils impliquent. (d'après Lécuyer, 2011) ${ }^{(*)}$

\begin{tabular}{|l|l|l|}
\hline $\begin{array}{l}\text { Niveaux de représentation } \\
\text { (débute) }\end{array}$ & Capacités observées & Capacités inférées \\
\hline $\begin{array}{l}\text { Analogique } \\
\text { (au fur et à mesure de la } \\
\text { maturation des sens) }\end{array}$ & Habituation & $\begin{array}{l}\text { Stockage en mémoire de l'information } \\
\text { Différenciation }\end{array}$ \\
\hline $\begin{array}{l}\text { Relationnelle } \\
\text { (vers la fin de la vie foetale) }\end{array}$ & Conditionnement & $\begin{array}{l}\text { Différence moi non-moi } \\
\text { Repérage des cooccurrences } \\
\text { Repérage des régularités }\end{array}$ \\
\hline $\begin{array}{l}\text { Transmodale } \\
\text { (à la naissance) }\end{array}$ & Transfert intermodal & $\begin{array}{l}\text { Organisation de la perception } \\
\text { de manière multimodale } \\
\text { Développement du repérage } \\
\text { des cooccurrences } \\
\text { Début de la construction de l'objet } \\
\text { Début de la capacité à extraire } \\
\text { des invariants }\end{array}$ \\
\hline $\begin{array}{l}\text { Abstraite } \\
\text { (vers 2 mois) }\end{array}$ & $\begin{array}{l}\text { Unité de l'objet } \\
\text { Permanence } \\
\text { Catégorisation } \\
\text { Perception de } \\
\text { représentations 2D }\end{array}$ & $\begin{array}{l}\text { Perception de l'objet à partir } \\
\text { d'informations partielles } \\
\text { Représentation de l'objet absent } \\
\text { Extraction d'invariants plus complexes }\end{array}$ \\
\hline
\end{tabular}

(*) Appelée ici «perceptive ». 
Les auteurs de cet article souhaitant se placer dans une perspective qualifiée de néoconstructiviste, cette idée de niveaux de représentation n'est pas sans rappeler celle de stades. Disons donc clairement qu'il ne s'agit pas ici de décrire une structure globale d'organisation de la pensée, mais simplement de tenir compte de ce que l'on sait sur le développement des capacités sensorielles et cognitives en fonction de l'âge et des conditions environnementales dans lesquelles est placé le fœtus, puis le bébé, ainsi que de permettre des rapprochements entre des performances présentes au même moment dans différents domaines de la connaissance. Les niveaux ainsi isolés, pour la commodité de la description du chercheur, n'excluent pas un développement continu, pas plus qu'ils n'excluent que des prémisses des capacités représentationnelles décrites à un niveau ne soient présentes au niveau précédent. Enfin, il n'est pas à exclure que des arguments nouveaux puissent conduire à proposer d'autres appellations pour tel ou tel niveau, d'autres niveaux ou au contraire à supprimer des distinctions de niveaux qui pourraient dans l'avenir se révéler artificielles.

Lécuyer (2011) distingue quatre niveaux, le premier permettant de rapporter l'habituation et donc la possibilité d'acquérir seulement des connaissances factuelles (représentation analogique), le deuxième rendant compte du conditionnement et donc de la possibilité de saisir des relations causales (représentation relationnelle), le troisième rapportant l'utilisation coordonnée des informations provenant de toutes les modalités sensorielles et permettant donc de commencer à parler d'objet, en ne réduisant pas celui-ci à l'information visuelle qui en est issue (représentation transmodale, que nous appellerons ici représentation perceptive) et le quatrième permettant d'extrapoler à partir d'une situation informationelle incomplète et permettant donc la permanence de l'objet (représentation abstraite). Un cinquième niveau, celui de la représentation symbolique, serait acquis plus tard.

Caractérisons un peu plus ces différents niveaux. Dès qu'une modalité sensorielle est fonctionnelle, elle est susceptible d'habituation. Un bon moyen de constater qu'elle est fonctionnelle est précisément de tester cette possibilité d'habituation. La preuve de l'habituation est apportée par la réaction à la nouveauté : réaugmentation des durées d'attention à la stimulation. Or, pour que cette réaction se produise, il faut qu'il y ait une différenciation entre le stimulus qui a fait l'objet de l'habituation et celui qui fait l'objet du test. C'est d'ailleurs la raison pour laquelle la méthode a été très productive dans les recherches sur le bébé : elle permet de connaître les capacités de différenciation. Mais pour différencier un stimulus présent d'un stimulus absent, il faut avoir une représentation de ce dernier. Cette représentation est donc une trace en mémoire du stimulus. C'est pourquoi, elle est appelée analogique et correspond à l'apprentissage par habituation. Ce premier niveau de représentation, nécessitant une maturité suffisante du système sensoriel impliqué, serait atteint à des âges différents (mais relativement proches) pour chacune des modalités sensorielles en fonction des décalages maturationnels. Il faut de plus noter qu'il ne s'agit pas d'apprentissage de règles mais de faits. 
Le caractère quelque peu restreint du monde dans lequel vit le fœtus présente l'avantage d'une grande répétitivité des situations de stimulation, ce qui facilite grandement la tâche d'un cerveau en pleine construction, ayant des capacités attentionnelles très limitées. En particulier, ceci facilite le repérage des cooccurrences ou des successions d'événements dans un délai suffisamment bref et dans un ordre constant. En d'autres termes, le fotus doit devenir capable d'apprentissages associatifs, donc de conditionnement. Quand peut-on considérer que des cooccurrences ou des successions rapides sont des phénomènes traités comme contingents? Précisément, quand un conditionnement est possible. Prenons l'exemple du simple et du double toucher : un fœtus qui touche la paroi placentaire reçoit une information tactile, alors que s'il touche son propre corps, il en reçoit deux. De plus, cette différence est en partie corrélée avec des informations proprioceptives : la position du bras n'est pas la même dans les deux cas, la contingence très élevée entre informations tactiles et proprioceptives doit permettre que la différenciation entre ce qui est et ce qui n'est pas le corps propre persiste même sans toucher. Ceci suppose un second niveau de représentation que Lécuyer (2011) qualifie de relationnel. Celui-ci apparaîtrait vers la fin de la vie fœtale, mais ne concernerait pas nécessairement de la même manière toutes les modalités sensorielles. En effet, si l'on prend l'exemple de l'audition, les sons atteignent bien l'oreille du bébé, mais les sources sonores sont situées en dehors de son champ perceptif autre que sonore, il semble donc difficile pour le fœtus de situer de manière précise ces stimulations en relation avec d'autres informations apportées par d'autres modalités : les sons n'arrivent que rarement en synchronie avec un événement non sonore. Citons, sans pouvoir préciser davantage la fréquence d'une telle opportunité, la possibilité que la mère danse sur une musique audible par le fœtus, ce qui peut permettre des synchronies entre sons et stimulations vestibulaires et tactiles. Le niveau de la représentation relationnelle se caractérise essentiellement par la capacité à faire des apprentissages associatifs sur la base d'un repérage des coïncidences ou des cooccurrences.

\section{$\underline{42}$}

La naissance va changer fondamentalement les conditions de relation entre les différentes modalités sensorielles, puisque la vision, modalité sensorielle dominante dans l'espèce humaine, entre réellement en jeu. Il en résulte nécessairement une réorganisation complète du système perceptif, et dès la naissance, le bébé tourne la tête vers une source sonore. Les correspondances entre les indices tactiles et visuels, qui permettent le transfert intermodal dès la naissance (Streri et Gentaz, 2004), facilitent certainement l'efficience rapide de la modalité visuelle. C'est dans ce contexte multimodal que sont acquises les premières connaissances postnatales : un nouveau-né ne reconnaît le visage de sa mère que si il l'a vue parler (Sai, 2005) et les temps de regard sont considérablement plus élevés si une information auditive accompagne le visuel que si les deux modalités sont stimulées séparément (Flom et Bahrick, 2007). Pour ces raisons, Lécuyer (2011) appelle transmodal le niveau de représentation qui suit la naissance. Ceci ne veut pas dire que des relations intermodales n'ont pas été établies avant la naissance, mais qu'en se structurant autour de la vision, l'intermodal constitue le passage de la sensation à la perception, c'est-à-dire à un niveau intégré d'organisation du réel. C'est pourquoi, nous préférons parler ici de représentation perceptive.

\section{$\underline{43}$}

Beaucoup de données montrent qu'aux environs de deux-trois mois, le bébé peut prendre en compte des propriétés des objets à un moment où celles-ci sont absentes des stimulations présentées. C'est bien sûr le cas des expériences sur l'unité de l'objet, ou sur la permanence de l'objet, mais c'est aussi le cas de celles qui portent sur la catégorisation : l'invariant qui 
définit une catégorie n'est lisible sur aucun des éléments de la catégorie, et doit être déduit de la série. L'application de cet invariant par les bébés qui traitent différemment deux stimulus nouveaux, parce que l'un appartient à la catégorie présentée et que l'autre n'y appartient pas (et est donc plus nouveau), relève bien du même niveau de représentation et est bien possible au même âge que la permanence de l'objet. C'est également au même âge qu'il devient capable de traiter des images comme ce qu'elles représentent, et donc de tenir compte là encore de propriétés absentes du stimulus.

Comprendre ce que représente une image, une photo ou encore comprendre l'action dans un film suppose la reconstruction d'une dimension absente de la stimulation visuelle : la dimension de profondeur. L'image est en effet par nature bidimensionnelle, et l'observateur va devoir utiliser des indices picturaux qui représentent la profondeur pour voir par exemple sur un écran qu'un objet est en train de passer derrière un autre objet. Afin de savoir comment les bébés comprennent les images, Durand et Lécuyer, (2002) ; Lécuyer et Durand, (1998) ont réexaminé la question de la permanence de l'objet de type Baillargeon, en proposant non pas des objets réels comme c'est classiquement le cas (Baillargeon et Graber, 1987 ; Baillargeon, Spelke et Wasserman,1985) mais des situations animées sur un écran de télévision. Avec la méthode de transgression des attentes, les auteurs ont cherché à déterminer si les bébés de quatre mois assignent à l'objet représenté les mêmes propriétés qu'à l'objet réel, en particulier son existence permanente. Les résultats montrent qu'avec un certain décalage, les connaissances des bébés sur l'objet réel semblent pouvoir s'appliquer à l'objet représenté. A 4 mois, les bébés sont surpris de voir qu'un « objet » caché cesse d'exister, mais cette compréhension reste initialement fragile puisque les bébés réagissent à l'objet disparu uniquement si les indices picturaux représentant la profondeur sont nombreux. Lorsque la profondeur est représentée par un seul indice (l'interposition), les bébés ne semblent pas comprendre la situation, lorsque la profondeur est représentée par plusieurs indices (l'interposition, la perspective, le gradient de texture), les bébés comprennent qu'un objet a disparu derrière un autre objet. C'est donc à partir de 3-4 mois que le bébé apprend l'usage des indices visuels picturaux pour se représenter la profondeur. La compréhension de l'image ne fonctionne pas en tout ou rien, mais se développe progressivement grâce à la prise en compte d'informations qui ne sont pas directement présentes dans la stimulation. Lécuyer (2011) propose d'appeler le niveau de représentation nécessaire à ces connaissances, représentation abstraite.

\section{Le passage d'un niveau de représentation au suivant}

La hiérarchie hypothétique de niveaux de représentation évoquée ci-dessus ne suffit pas à rendre compte du développement des capacités cognitives lui-même nécessaire au développement des connaissances. Il faut faire l'hypothèse supplémentaire de quelques capacités qui vont permettre de passer d'un niveau de représentation au niveau supérieur. 
Le niveau analogique suppose une capacité de stockage des informations sensorielles et de comparaison des informations stockées aux informations actuelles permettant la discrimination, c'est ce que supposent les modèles d'habituation, comme par exemple celui de Cohen (1972) qui est l'un des plus connus.

\section{$\underline{47}$}

Passer de ce premier niveau au second : la représentation relationnelle nécessite une capacité à repérer des régularités, dans la coïncidence ou la succession rapide des événements.

Michotte (1962) s'est opposé à Piaget sur la question de la perception de la causalité. Si pour ce dernier, la causalité est le résultat d'une lente construction, pour Michotte, il s'agit d'une gestalt innée. Les recherches de cet auteur sur la perception de la causalité chez l'adulte lui ont permis de mettre en évidence un phénomène du plus grand intérêt. Si un carré mobile $\mathrm{A}$ heurte un carré fixe $B$ et que $B$ démarre instantanément, les adultes décrivent l'événement en termes causaux (A a poussé B). Si un délai court, mais perceptible et perçu, sépare le choc du départ de B ou si il n'y a pas réellement contact, mais qu'un espace court mais perçu sépare A de $\mathrm{B}$ au moment du départ de $\mathrm{B}$, les adultes continuent à percevoir ces événements comme causaux. La causalité perçue résiste donc à la logique.

\section{$\underline{48}$}

Dans des situations plus complexes, la coïncidence ou la corrélation sont souvent interprétées dans un sens causal dans la pensée naturelle : la démarche scientifique consiste en grande partie à séparer coïncidence et cause. Il est donc possible que le système de repérage évoqué ci-dessus, rapidement automatisé pour être efficace, soit la base de ces formes de pensée naturelle. Ce qui est donc supposé ici est l'existence extrêmement précoce d'un détecteur de contingence donnant une interprétation de type causal à toute coïncidence et toute succession rapide d'événements, même quand cette contingence est illusoire. Un mécanisme explicatif de la connaissance de lois chez le bébé doit en effet servir à expliquer aussi bien les fausses que les vraies, ces dernières n'étant pas les plus nombreuses.

\section{$\underline{49}$}

Passer du niveau relationnel au niveau perceptif ne suppose pas de capacités cognitives spécifiques, mais que malgré l'hétérogénéité complète des supports physiques des sensations et donc de l'anatomie et de la physiologie des différents récepteurs sensoriels, les représentations se construisent à un niveau suprasensoriel de manière extrêmement précoce. C'est pourquoi, ce passage est parfois considéré comme l'accès véritable à la perception. Il est intéressant de rapprocher ce changement de niveau des propositions de Mounoud (passage du sensorimoteur au perceptivo-moteur). Les données allant dans ce sens ne manquent pas dans la littérature (Streri et Gentaz, 2004 ; Sai, 2005). Cette importance de la multimodalité n'a d'ailleurs rien d'étonnant dans la mesure où elle est une source de complémentarité dans l'information, les différentes modalités sensorielles apportant des informations de nature différente et d'organisation spatiotemporelle différente. Elle est en même temps une source de redondance puisque ces informations peuvent en partie se recouper. Elle est enfin source de repérage de contingences. 
Le passage au niveau abstrait est basé sur un processus d'extrapolation, puisqu'il permet de tenir compte de propriétés du monde qui ne font pas partie des stimulations présentes. Là encore, un rapprochement avec la pensée naturelle est informatif puisque les extrapolations y sont fréquentes et souvent abusives. Un tel mécanisme permet aisément de transformer une régularité en " règle », ou pour le moins de produire des attentes concernant les événements possibles, probables, nouveaux mais imaginables, surprenants ou strictement impossibles, avec, chez le bébé comme chez l'adulte, des durées de traitement qui augmentent avec l'étrangeté. Contrairement à Mounoud, nous n'appelons pas ce niveau « cognitif », d'une part parce que la perception est une activité cognitive, d'autre part parce que nous centrons l'appellation précisément sur la prise en compte de ce qui n'est pas perceptible, et donc la nécessité d'un processus d'abstraction.

\section{$\underline{51}$}

Les capacités ci-dessus inférées font partie intégrante des systèmes de fonctionnement cognitifs tels qu'ils se mettent en œuvre dès leurs débuts. En d'autres termes et pour reprendre un vocabulaire classique, ils sont supposés innés. Pour autant, cette position se sépare radicalement du nativisme sur deux points majeurs. Le premier est que ce qui est supposé génétiquement déterminé n'est pas un ensemble de connaissances, donc un ensemble de propriétés du monde, mais un ensemble de capacités fonctionnelles du sujet. En ce sens, cette position se rapproche de la perspective de Mounoud (1979). Le second est que si les deux modèles servent à expliquer les « lois » de fonctionnement du monde que comprennent les bébés de 2-3 mois, le nativisme suppose un mode de fonctionnement dans lequel toutes les « lois » connues des bébés sont vraies, alors que le présent point de vue vise à expliquer la construction de lois en général et donc la généralisation justifiée aussi bien que la généralisation abusive. Notre proposition est donc à la fois plus parcimonieuse et plus générale que ne l'est le nativisme.

\section{L'être humain aux sources de la connaissance}

Si dans le contexte de la théorie nativiste, comme dans le contexte piagétien, le bébé a longtemps été envisagé seul face à son monde physique, ces dernières années, la multiplication des travaux sur le rôle du milieu social dans lequel évolue et interagit le bébé n'a fait que rendre son importance à cette évidence : l'espèce humaine est une espèce sociale et cette caractéristique est d'autant plus importante que le sujet est plus jeune.

\section{$\underline{53}$}

On l'oublie souvent mais il est important de rappeler que les premières formes de prise de contact du nouveau-né humain avec son nouvel environnement sont avant tout des contacts humains et non des relations avec le monde des objets. Durant les mois qui suivent la naissance, les situations d'éveils d'un bébé sont principalement des situations de face-à-face avec son entourage familial. Paradoxalement on l'a vu, les recherches ont surtout porté sur la connaissance du monde physique, appréhendé avec la seule modalité visuelle. Le postulat implicite était que la source d'information essentielle du bébé se trouve dans le monde 
physique. Pourtant, le monde social est la meilleure forme de stimulation possible pour un bébé. C'est donc l'observation active du monde social qui permet d'apprendre les lois du fonctionnement du monde physique, lois auxquelles le vivant est lui aussi soumis, même s'il peut produire des réactions : un adulte sans support tombe, comme tout autre objet. Une main ne peut pas plus traverser un objet qu'une boîte, etc.

\section{$\underline{54}$}

Mais de plus, le monde social est capable de tenir compte des caractéristiques spécifiques du sujet et de s'adapter à ses capacités sensorielles et attentionnelles. Lorsque l'on regarde des adultes ou des enfants d'âge scolaire face à des bébés, on peut constater qu'ils ont des comportements complètement spécifiques (Papoušek et Papoušek, 2002). Ces formes de parentage intuitif consistent à produire des stimulations adaptées aux capacités sensorielles des bébés (exagération des expressions faciales, décalages de la voix vers l'aigu, lenteur des mouvements et des productions sonores) et à produire beaucoup de redondance intra et intersensorielle. Mais surtout, l'organisation temporelle de la stimulation est adaptée aux capacités attentionnelles des bébés, et s'il apparaît que les contingences liées à un événement n'ont pu être perçues de manière efficace, cet événement est répété autant que nécessaire.

\section{$\underline{55}$}

Le bébé ne produit pas les changements de l'environnement nécessaires à sa compréhension, mais l'adulte les produit pour lui. Donc à un premier niveau, celui de la stimulation, rien n'est plus informatif sur les caractéristiques du monde physique que le monde social. Si l'on ajoute les expériences vécues in utero qui guident le nouveau-né, on comprend pourquoi de toutes les stimulations perçues, le nouveau-né se montre d'emblée plus attiré par les stimulations humaines ou produites par des humains, et ce quelle que soit la modalité envisagée (préférence visuelle pour des visages ou des mouvements produits par des humains, préférence auditive pour des sons de parole ou des voix humaines, préférence olfactive pour des odeurs humaines ...). Les mécanismes d'apprentissages s'exercent d'abord et avant tout dans un contexte social. Les bébés expérimentent auprès de leur mère (ou de leur propre corps) pour généraliser ensuite à d'autres contextes, ce qui pourrait expliquer une certaine forme d'animisme dans la pensée naturelle.

\section{$\underline{56}$}

Dans cette perspective, les parents ont un rôle fondamental : ils offrent aux nourrissons les conditions idéales pour leur permettre d'apprendre par des ajustements tout à fait adaptés au niveau de développement. A partir de trois mois, les échanges du bébé avec ses parents s'étendent aux objets. On peut remarquer qu'en interagissant, les parents donnent naturellement des leçons sur les objets : de manière régulière, ils font des démonstrations qui permettent au bébé de comprendre le fonctionnement des objets (Rochat, 2006). Il est donc indispensable pour comprendre le développement cognitif du nourrisson d'observer la manière dont fonctionnent les relations de celui-ci avec cet environnement social.

\section{$\underline{57}$}

Une dernière remarque concernant l'environnement social : il est par nature multisensoriel. Il véhicule une multitude d'informations visuelles, sonores, tactiles, olfactives qui stimulent de manière régulière et synchrone dans le temps l'ensemble des entrées sensorielles. Il est 
remarquable que le bébé évolue dans un environnement lui fournissant des conditions idéales pour percevoir des régularités. Un grand nombre de recherches a confirmé l'adaptation du bébé à cette complexité multimodale (Bahrick et Lickliter, 2002 ; Lewkowicz, 1994). Non seulement, les prises d'informations sont cohérentes dès la naissance et permettent des mises en relation mais elles paraissent également essentielles pour apprendre sur l'environnement physique et social. Actuellement, la reconnaissance d'un visage par le nouveau-né de quelques heures s'explique par une mise en relation entre des informations visuelles nouvelles (le visage) et des informations auditives (la voix humaine) et n'est pas observée sans cette mise en relation (Sai, 2005 ; Coulon, Bahia et Streri, 2011). La perception multisensorielle permet le repérage des synchronies temporelles et spatiales et organise l'attention des bébés (initialement sur la base d'informations redondantes, c'est-à-dire répétées à travers plusieurs modalités). Quelques mois plus tard, seuls les sons de parole vont pouvoir jouer ce rôle. C'est ce dont cherche à rendre compte le modèle de la redondance intersensorielle (Bahrick et Licklikter, 2002) : la perception multisensorielle conditionne dans une large mesure les apprentissages. Le bébé de trois mois n'est pas toujours en situation de repérer les contingences qui se produisent dans son environnement physique et après la naissance, le développement de la représentation relationnelle dépend des possibilités de repérage de ces contingences, lesquelles sont bien plus souvent fournies par le milieu social. En l'état actuel de nos connaissances, il devient nécessaire de poser la question de la multimodalité, ou plutôt des multimodalités en tant que processus/mécanismes développementaux à l'origine des premières connaissances. Le fonctionnement intersensoriel est avéré dès (avant ?) la naissance et se caractérise par une grande variété de relations. Certaines de ces relations, parce que redondantes et synchrones dans le temps, canalisent l'attention du bébé, d'autres, parce que provenant d'une même source dans l'espace, lui permettent de construire une représentation unifiée d'un objet. Un enjeu pour l'avenir sera de préciser l'ensemble des relations intersensorielles ainsi que le rôle des différentes modalités dans les apprentissages précoces tout en tenant compte de la très probable redistribution des rôles de chaque modalité avec l'entrée en jeu de la vision. Il en va de même pour la représentation abstraite, base de la permanence de l'objet. Celle-ci est à peu près toujours considérée du seul point de vue de la vision, ce qui est légitimé par le fait que la plupart des objets physiques absents dans la modalité visuelle le sont aussi dans les autres modalités. Mais quand une mère sort du champ visuel du bébé, ou bien elle est en contact tactile avec lui (par exemple : elle le met sur le ventre et il ne peut la voir) ou bien elle s'éloigne et n'a de contact ni visuel ni tactile, ni odorant. Alors, immanquablement, elle lui parle. Il est donc possible à un bébé d'apprendre qu'un objet absent dans une modalité peut être présent dans une autre et que les passages, d'une modalité à une autre ou de trois modalités à deux ou à quatre concernant l'information venue du même objet, se font très vite et en permanence. On peut difficilement imaginer meilleures conditions pour apprendre que le passage d'une modalité à zéro ne signifie pas la fin de l'existence de l'objet. Il s'agirait donc là du passage de la représentation perceptive à la représentation abstraite.

\section{De quelques conséquences de ce qui précède}

Si l'on confronte les positions théoriques actuellement défendues par différents chercheurs pour expliquer les premières connaissances et l'image du bébé que chaque théorie dessine, et donc indirectement l'image de l'action éducative des parents qui en résulte, apparaît une situation paradoxale. En effet, la théorie dominante et la mieux connue dans le public est le 
nativisme, mais la popularisation de celui-ci semble avoir développé le marché de la stimulation précoce. Pourtant, si l'essentiel du savoir est connu à la naissance, il ne sert à rien d'essayer d'apprendre ! L'innéisme classique de Gesell l'avait d'ailleurs conduit à un grand libéralisme éducatif.

\section{$\underline{59}$}

La raison de ce paradoxe est sans doute la conjonction, peu pensée d'un point de vue théorique, entre l'augmentation considérable de l'importance sociale de la réussite scolaire, le développement de l'idée que les premières années de la vie sont déterminantes (le vieux « tout se joue avant six ans » aisément transformé en " avant six mois ») et la découverte de connaissances précoces. Ajoutons hélas la découverte de la mort neuronale, processus anatomophysiologique normal dans la période périnatale, que certaines bonnes âmes ont voulu éviter au nouveau-né par la stimulation adéquate ! L'utilisation de données neurologiques mal digérées est d'ailleurs une constante chez les marchands de stimulation. Le meilleur exemple est la spécialisation hémisphérique, caricaturée par la production de stimulations « qui s'adressent au cerveau gauche » (ou au cerveau droit). Il est donc intéressant de voir ce que l'on peut penser de cette question du point de vue des différents courants théoriques évoqués ci-dessus.

Dans une perspective piagétienne, il n'est de savoir que reconstruit et l'activité du sujet est la condition sine qua non de tout apprentissage. C'est bien l'un des points sur lesquels Piaget fait l'économie du social : le sujet est fondamentalement seul face à la connaissance à acquérir. "On n'apprend jamais rien des maîtres qu'en reconstruisant leur pensée, à défaut de quoi elle ne se fixe ni dans l'intelligence ni même dans la mémoire » (Piaget, 1974) Une pédagogie piagétienne des premières connaissances ne conduit donc pas à la surstimulation.

Dans une perspective nativiste, il n'y a guère d'apprentissages importants à faire, puisqu'il y a donc impossibilité d'apprendre ce qu'on ne possède pas déjà sous une forme ou sous une autre (Mehler, 1983). Dans une perspective nietzschéenne, le bébé n'a qu'à devenir ce qu'il est.

Il en va tout autrement dans la perspective défendue ici, puisqu'elle met en avant le rôle capital du milieu social dans le développement. Puisque tout le monde est d'accord sur l'idée d'un bébé actif dans ses apprentissages, il reste à tirer toutes les conséquences de cet aspect de son développement. C'est le cas quand l'attitude éducative a pour premier objectif de placer le nourrisson dans les conditions qui lui permettent d'explorer activement son monde. Ce n'est pas le cas quand on cherche simplement à le stimuler. Dans la perspective que nous défendons, le bébé possède à chaque étape un potentiel de développement (un contexte, des systèmes prêts à recueillir l'information, des capacités pour mémoriser : connaître et reconnaître ...) mais il est nécessaire qu'il utilise activement ce potentiel pour la suite de ce développement : il lui faut être ce qu'il devient. 
Finalement, nous pouvons souligner à quel point les recherches actuelles sur la cognition du petit enfant sont en perpétuel renouvellement, mais elles se rejoignent sur un point : la nécessité de dépasser le cadre étroit du nativisme pour tenter de comprendre et expliquer les mécanismes les plus précoces de la pensée et de son développement. On le voit aujourd'hui, le croisement des données expérimentales issues des différentes disciplines conduit à une description de plus en plus complexe des processus d'apprentissage et, en conséquence, à une interprétation théorique qui s'éloigne de plus en plus des hypothèses nativistes monocausales et déterministes. Leur succès est sans doute lié à l'avantage de correspondre aux canons de la conception spontanée des relations causales et d'offrir une vision du réel simple à assimiler. Bien que ce type de considération doive toujours être fait avec prudence, il semble donc que nous soyons actuellement dans une phase d'instabilité théorique et de redistribution des cartes. Les conclusions que l'on peut tirer de l'analyse qui précède, si elles se caractérisent par la nécessité d'introduire de la complexité là où l'on aurait aimé faire simple, seront sans doute rapidement dépassées et critiquées pour cause de simplisme. Comme William James il y a un siècle, puis comme Piaget, les chercheurs actuels restent en effet confrontés à la question de savoir comment des formes d'organisation émergent d'un amas de cellules indifférenciées et par quels chemins la dynamique interne de ce système et l'environnement arrivent à exercer leur indissociable influence.

\section{Références}

- EN LIGNe AGUIAR A., BAILLARGEON R. : «2.5-Month-old infants’ reasoning about when objects should and should not be occluded », Cognitive Psychology, 1999 ; 39 (2) : 116-157.

- BAHRICK L.E., LICKLITER R. : «Intersensory redundancy guides early perceptual and cognitive development », in : KAIL R.V., (ed.), Advances in child development and behavior, Academic Press, San Diego, 2002, pp. 153-187.

\section{EN LIGNE BAHRICK L.E., HERNANDEZ-REIF M., FLOM R. : « The} development of infant learning about specific face-voice relations », Developmental Psychology, 2005 ; 41(3) : 541-552.

- EN LIGNE BAHRICK L.E., LICKLITER R., FLOM R. : « Intersensory redundancy guides the development of selective attention, perception, and cognition in infancy », Current Directions in Psychological Science, 2004 ; 13 (3) : 99-102.

- EN LIGNe BAILlARGEON R., WANG S. : « Event categorization in infancy », Trends in Cognitive Sciences, 2002 ; 6(2) : 85-93.

- BAILLARGEON R. : "Le raisonnement des bébés à propos des objets cachés », in : LÉCUYER R., (ed.), Le développement du nourrisson, Dunod, Paris, 2004, pp. 271308.

- BAILLARGEON R., GRABER M. : «Where's the rabbit ? 5.5-month-old infants' representation of the height of a hidden object », Cognitive Development, $1987 ; 2$ (4) : 375-392.

- en ligne BAILlaRGEON R., SPELKE E.S., WASSERMAN S. : « Object permanence in five-month-old infants », Cognition, $1985 ; 20$ (3) : 191-208.

- EN LIGNE BEHL-CHADHA G. : «Basic-level and superordinate-like categorical representations in early infancy », Cognition, 1996 ; $60: 105-141$. 
- EN LIGNE BERLYNE D.E. : « The influence of complexity and novelty in visual figures on orienting responses », Journal of Experimental Psychology, 1958 ; 55 : 289-296.

- En ligne BOGARTZ R.S., SHINSKEY J.L., SCHILLING T.H. : « Object permanence in five-and-a half-month-old infants ? », Infancy, $2000 ; 1$ (4) : 403-428.

- EN LIGNE BORNSTEIN M.H., MASH C. : « Experience-based and on-line categorization of objects in early infancy », Child Development, 2010 ; 81 (3) : 884897.

- EN LIGNE BORNSTEIN M.H., SIGMAN M.D. : « Continuity in mental development from infancy », Child Development, $1986 ; 57: 251-274$.

- BULLINGER A. : "Elaboration cognitive des conduites sensori-motrices », in : BUTTERWORTH G., (ed.), Infancy and epistemology, Harvester Press Cohen, London, (1972), 1982.

- ASHON C.H., COHEN L.B. : « Eight-month-old infants' perceptions of possible and impossible events », Infancy, $2000 ; 1$ (4) : 429-446.

- EN LIGNE COHEN L.B. : «Attention-getting and attention-holding processes of infant visual preferences », Child Development, $1972 ; 43: 869-879$.

- COULON M., BAHIA G., STRERI A. : «Recognition of unfamiliar talking faces at birth », International Journal of Behavioral Development, sous presse, 2011.

- EN LIGNE DECASPER A.J., FIFER W.P. : « On human bonding : Newborn prefer their mothers' voices », Science, $1980 ; 208: 1174-1176$.

- DECASPER A.J., SPENCE M. : «Prenatal maternal speech influences newborns“ perception of speech sounds », Infant Behavior \& Development, 1986 ; 9 (2) : 133150.

- EN LIGNE DEL GIUDICE M. : « Alone in the dark ? Modeling the conditions for visual experience in human fetuses », Developmental Psychobiology, $2011 ; 53$ (2) : 214-219.

- EN LIGNE DURAND K., LÉCUYER R. : « Object permanence observed in four month-old-infants with a 2D display », Infant Behavior \& Development, $2002 ; 25$ (3) : 269-278.

- EN LIGNE DURAND K., LECUYER R., FRICHTEL M. : « Representation of the third dimension : The use of perspective cues by 3 - and 4-month-old infants », Infant Behavior \& Development, 2003 ; 26 (2) : 151-166.

- EN LIGNE EIMAS P.D., QUINN P.C. : « Studies on the formation of perceptually based basic-level categories in young infants », Child Development, 1994 ; 65 : 903917.

- EN LIGNE EIMAS P.D., QUINN P.C., COWAN P. : « Development of exclusivity in perceptually based categories of young infants », Journal of Experimental Child Psychology, 1994 ; 58 (3) : 418-431.

- EN LIGNE FAGAN J.F., HOLLAND C.R., WHEELER K. : « The prediction, from infancy, of adult IQ and achievement », Intelligence, 2007 ; 35 (3) : 225-231.

- FANTZ R.L. : «Pattern vision in young infants », Psychological Record, 1958 ; 58 : 43-47. 
- EN LIGNE FLOM R., BAHRICK L.E. : «The development of infant discrimination of affect in multimodal and unimodal stimulation : The role of intersensory redundancy », Developmental Psychology, 2007 ; 43 (1) : 238-252.

EN LIGNE FURRER S.D., YOUNGER B.A. : « Beyond the distributional input? A developmental investigation of asymmetry in infants' categorization of cats and dogs », Developmental Science, 2005 ; 8 (6) : 544-550.

- GRANIER-DEFERRE C., SCHAAL B., DECASPER A.J. : « Les prémisses fotales de la cognition », in : LECUYER R. (ed.), Le développement du nourrisson, Dunod, Paris, 2004.

- HESPOS S.J., BAILLARGEON R. : « Reasoning about containment events in very young infants », Cognition, $2001 ; 78$ (3) : 207-245.

- EN LIGNE JOHNSON S.P., NAÑEZ J.E. : «Young infants' perception of object unity in two-dimensional displays », Infant Behavior \& Development, 1995 ; 18 : 133143.

- EN LIGNE KAVSEK M. : « Predicting later IQ from infant visual habituation and dishabituation : A meta-analysis », Journal of Applied Developmental Psychology, $2004 ; 25$ (3) : 369-393.

- EN LIGNE KELLMAN P.J., SPELKE E.S. : « Perception of partly occluded objects in infancy », Cognitive Psychology, 1983 ; 15 : 483-524.

- $\quad$ LECANUET J.P., GRANIER-GEFERRE C., SCHAAL B. : " La continuité sensorielle transnatale ", in : POUTHAS V., JOUEN F., (eds), Les comportements $d u$ bébé : expression de son savoir?, Mardaga, Liège, 1993, pp. 33-51.

- EN LIGNE LÉCUYER R., DURAND K. : «Bi-dimensional representations of the third dimension and their perception by infants », Perception, $1998 ; 27: 465-472$.

- EN LIGNE LÉCUYER R. : «Habituation and attention, novelty and cognition : Where is continuation ? », Human Development, 1989a ; 32 : 148-157.

- LÉCUYER R. : «Bébés astronomes, bébés psychologues. L’intelligence de la première année », Mardaga, Bruxelles, 1989b.

- LÉCUYER R. : «Développement continu ou développement par stades : l'exemple du nourrisson », in : Hommage à César Flores. L'Harmatan, Paris, 1994a.

- LÉCUYER R. : «On the beginnings of intellectual development », in : MONTANGERO J., CORNU-WELLS A., TRIPHON A., VONÈCHE J., (eds.), Conceptions of change over time. Cahiers de la fondation Archives Jean Piaget $N^{\circ}$ 13., Archives Jean Piaget, Genève, 1994b.

- LÉCUYER R. : « Les sources de la connaissance », in : LÉCUYER R., STRERI A., PECHEUX M.G., (eds.), Psychologie cognitive du nourrisson. Tome 2., Nathan, Paris, 1996, pp. 127-181.

- LÉCUYER R. : « De la pensée à l'acte », in : HOUDE O., MELJAC C., (eds.), L'esprit piagétien, Presses Universitaires de France, Paris, 2000, pp. 89-98.

- LÉCUYER R. : «Rien n'est jamais acquis. De la permanence de l'objet... de polémiques », Enfance, $2001 ; 53$ (1) : 35-65.

- LÉCUYER R. : «Faut-il ajouter le calcul aux capacités cognitives précoces du nourrisson, qui se multiplient, ou peut-on se soustraire à cette hypothèse qui divise ? », Intellectica, $2002 ; 34$ (1) : 231-251.

- LÉCUYER R. : «La représentation va pouvoir commencer », in : LÉCUYER R., (ed.) Le développement du nourrisson, Dunod, Paris, 2004, pp. 271-308. 
- EN LIGNe LÉCUYER R. : «Sans mobile apparent », Enfance, 2011 ; 63 (1) : 1736.

- LÉCUYER R., POIRIER C. : «Categorization of geometric figures composed of four elements by 5.5-month-old infants », Cahiers de Psychologie Cognitive/Current Psychology of Cognition, 1994 ; 13 (4) : 493-509.

- LÉCUYER R., TRERI A. : «Information intake in infants : Links between visual and tactual habituation », Cahiers de Psychologie Cognitive, 1986 ; 6 (6) : 565-574.

- LÉCUYER R., RIVIÈRE J., DURAND K. : «The sources of spatial cognition », in : AURNAGUE M., HICKMANN M., VIEU L., (eds.), The categorization of spatial entities in language and cognition, Benjamins, Amsterdam, 2006, pp. 247-266.

- LEMAINE G., MATALON B. : Hommes supérieurs, hommes inférieurs?, Armand Colin, Paris, 1985.

- LEWKOWICZ, DJ. : «Development of intersensory perception in human infants », in : LEWKOWICZ D.J., LICKLITER R., (eds.), Development of intersensory perception : Comparative perspectives, Lawrence Erlbaum Associates, Norwood, 1994.

- EN LIGNE MANDLER J.M. : «How to build a baby : On the development of an accessible representational system », Cognitive Development, 1988 ; 2 : 113-136.

- MANDLER J.M. : «How to build a baby : II. Conceptual primitives », Psychological Review, 1992 ; 99 (4) : 587-604.

- EN LIGNE MANDLER J.M. : « Babies think before they speak », Human Development, $1998 ; 41$ (2) : 116-126.

- MEHLER J., DUPOUX E. : Naître Humain, Odile Jacob, Paris, 1990.

- MEHLER J. : « La connaissance avant l'apprentissage », in : DE SCHONEN S., (ed.), Le développement dans la première année, Presses Universitaires de France, Paris, 1983.

- MiCHOTTE A. : Causalité, permanence et réalité phénoménales », Publications Universitaires de Louvain, Louvain, 1962.

- MOUNOUD P. : «Développement des systèmes de représentation et de traitement chez l'enfant », Bulletin de Psychologie, $1971 ; 296$ : 261-272.

- MOUNOUD P. : «Les révolutions psychologiques de l'enfant », Archives de Psychologie, $1976 ; 171: 103-114$.

- MOUNOUD P. : «Développement cognitif : construction de structures nouvelles ou construction d'organisations internes », Bulletin de Psychologie, 1979 ; 343 : 107-118.

EN LIGNE NEEDHAM A., BAILLARGEON R. : « Intuitions about support in 4.5month-old infants », Cognition, $1993 ; 47$ (2) : 121-148.

- PAPOUSEK H., PAPOUSEK M. : «Intuitive parenting », in : BORNSTEIN M.H., (ed.), Handbook of parenting : Vol. 2 : Biology and ecology of parenting (2nd ed.), Lawrence Erlbaum Associates Publishers, Mahwah, 2002, pp. 183-203.

- PIAGET J. : Réussir et comprendre, Presses Universitaires de France, Paris, 1974.

- EN LIGNE POIRIER C. : «L'abstraction d'une représentation centrale chez les bébés de trois mois », L'Année Psychologique, 1995 ; 95 (3) : 377-400.

- POIRIER C., LÉCUYER R., CYBULA C. : «Categorization of geometric figures composed of three or four elements by 3-month-old infants », Cahiers de Psychologie Cognitive/Current Psychology of Cognition, 2000 ; 19 (2) : 221-244.

- $\quad$ RAKINSON D.H., OAKES L.M. (eds.), Early category and concept development. Making sense of the blooming, buzzing confusion, Oxford University Press, Oxford, 2003. 
- EN LIGNE RIVIERE J., LECUYER R. : « The C not B error : A comparative study », Cognitive Development, $2003 ; 18$ (3) : 285-297.

- ROCHAT P. : Le monde des bébés, Odile Jacob, Paris, 2006.

- EN LIGNE SAIF.Z. : "The role of the mother's voice in developing mother's face preference : Evidence for intermodal perception at birth », Infant and Child Development, $2005 ; 14: 29-50$.

- SCHAAL B., GOUBET N., DELAUNAY-EL ALLAM M. : « Concordances et discordances entre sensorialité et écologie néonatales : attentes sensorielles et réponses adaptatives chez l'enfant prématuré », in : MELLIER D., (ed.), Le développement du nouveau-né prématuré, Solal Editeur, Marseille, 2011.

- en LIGNe SCHAAL B., HUMMEL T., SOUSSIGNAN R. : «Olfaction in the fetal and premature infant : Functional status and clinical implications », Clinics in Perinatology, 2004 ; $31: 261-285$.

- en ligne SCHAAL B., MARLIER L., SOUSSIGNAN R. : « Human foetuses learn odours from their pregnant mother's diet », Chemical Senses, $2000 ; 25: 729$ 737.

- EN LIGNE SCHAAL B., MARLIER L., SOUSSIGNAN R. : « Neonatal responsiveness to the odour of amniotic fluid », Biology of the Neonate, $1995 ; 67$ : 397-406.

- EN LIGNE SCHAAL B., MARLIER L., SOUSSIGNAN R. : « Olfactory function in the human fetus : Evidence from selective neonatal responsiveness to the odor of amniotic fluid », Behavioral Neuroscience, 1998 ; 112:1438-1449.

- EN LIGNe MARLIER L., CHAAL B. : «Human newborns prefer human milk: Conspecific milk odor attractive without postnatale exposure », Child Development, $2005 ; 76: 155-168$.

- SCHILLING T.H. : «Infants' looking at possible and impossible screen rotations : The role of familiarization", Infancy, $2000 ; 1$ (4) : 389-402.

- En LIGNe SLATER A., JOHNSON S.P., KELLMAN P.J., SPELKE E.S. : «The role of three dimensional cues in infants' perception of partly occluded objects », Early Development and Parenting, 1994 ; 3 : 187-191.

- en LIGNe SLATER A., MORISON V., SOMERS M., MATTOCK A. : « Newborn and older infants' perception of partly occluded objects », Infant Behavior \& Development, $1990 ; 13$ (1) : 33-49.

- EN LIGNE SPELKE E.S. : «Initial knowledge : Six suggestions », Cognition, $1994 ; 50: 431-445$.

- SPELKE E.S. : « Nativism, empiricism, and the origins of knowledge », Infant Behavior \& Development, 1998 ; 21 (2) : 181-200.

- EN LIGNE STRERI A., GENTAZ E. : «Cross-modal recognition of shape from hand to eyes and handedness in human newborns », Neuropsychologia, $2004 ; 42$ (10) : 1365-1369.

- YOUNGER B.A. : «Parsing objects into categories : Infants' perception and use of correlated », in : D.H. RAKINSON, L.M. OAKES, (eds.), Early category and concept development. Making sense of the blooming, buzzing confusion, Oxford University Press, Oxford, 2003, pp. 77-102. 


\section{Notes}

[1]

Professeur Emérite, Université Paris Descartes LPCN. 71, avenue Edouard Vaillant, 92774 Boulogne-Billancourt cedex, France.

roger.lecuyer@parisdescartes.fr

[2]

Maître de Conférences, Université de Bourgogne. Centre des Sciences du Goût et de l'Alimentation, CNRS UMR 6265, INRA, Agrosup. 9E, boulevard Jeanne d'Arc, 21000 Dijon, France.

\section{karine.durand@u-bourgogne.fr}

\section{[3]}

Ces trois termes sont utilisés dans la littérature de manière équivalente. Nous les utiliserons donc indifféremment.

\section{Résumé}

Français

La révolution méthodologique des années 1950-60 a accru considérablement nos moyens d'étude des capacités cognitives du nourrisson. Cette révolution a entraîné des bouleversements dans les théories et en particulier mis en cause la théorie de Piaget. Cependant, deux types de démarches ont toujours été suivis en parallèle. Un certain nombre d'auteurs ont cherché à se situer dans une perspective post-Piagétienne et c'est leur point de vue qui sera d'abord abordé. D'autres auteurs ont au contraire mis au point un cadre théorique radicalement nouveau : le nativisme, et c'est ce courant, qui a dominé ce domaine de recherche, qui sera ensuite exposé. L'existence d'apprentissages prénatals a mis en difficulté le nativisme. C'est pourquoi, ces apprentissages seront ensuite évoqués. Mais si donc les apprentissages commencent très tôt, aucune connaissance de règle n'a pu être mise en évidence avant l'âge de deux à trois mois, ce qui est évidemment un échec pour le nativisme. Dans la période actuelle, il semble donc possible et nécessaire de revenir à une perspective théorique constructiviste, développementaliste et donnant toute sa place au milieu social, c'est-à-dire au contexte de vie quotidien du bébé. L'objectif de l'article est de présenter cette perspective. A cet effet, il propose un système de niveaux de représentation susceptibles de rendre compte de capacités jugées équivalentes et présentes dans une même tranche d'âge.

Mots-clés

- nourrisson et apprentissage précoces

- nativisme et néoconstructivisme

- représentations, multimodalité et environnement social 


\section{Plan de l'article}

1. Les premières connaissances : une place à part en psychologie?

2. Peut-on ne pas être nativiste ?

3. L'apprentissage commence avant la naissance

4. Les connaissances mises en évidence de deux à cinq mois sont-elles innées ?

5. A quelles conditions un néoconstructivisme est-il envisageable?

6. Des niveaux de représentation et des mécanismes d'apprentissage

7. Le passage d'un niveau de représentation au suivant

8. L'être humain aux sources de la connaissance

9. De quelques conséquences de ce qui précède

\section{Pour citer cet article}

Lécuyer Roger, Durand Karine, « Aux sources de la connaissance. L'état du débat constructivisme/ nativisme chez le très jeune enfant », Devenir 3/2012 (Vol. 24), p. 181-214 URL : www.cairn.info/revue-devenir-2012-3-page-181.htm.

DOI : $\underline{10.3917 / \mathrm{dev} .123 .0181}$. 